\title{
Prognostic Implication of Survivin Expression in Oral Squamous Cell Carcinoma_-An Immunohistochemical Study
}

\author{
M Ajithkumar ${ }^{1}$, Chinnakonda Raveendranath Murali $^{2}$, Nandimandalam Venkata Vani ${ }^{3}$
}

\begin{abstract}
Aim: Immunohistochemical expression of survivin was analyzed among the three histological grades (well differentiated, moderately differentiated, and poorly differentiated) of oral squamous cell carcinoma (OSCC).

Materials and methods: The study material consisted of 60 formalin-fixed paraffin-embedded tissue samples: 15 cases each of well, moderate, and poorly differentiated OSCC and normal oral mucosal (NOM) tissues as the control. Survivin expression was evaluated immunohistochemically and statistical analysis of data was performed using Fisher's Chi-square and analysis of variance (ANOVA) tests.

Results: Survivin was expressed in all grades of OSCC, but absent in normal oral tissue samples. Poorly differentiated OSCC exhibited 51 to $75 \%$ immunopositivity (53.3\%) and severe staining intensity (46.7\%) for survivin, predominantly in nuclear areas. While moderately differentiated OSCC had 26 to $50 \%$ immunopositivity (40\%) and moderate staining intensity ( $80 \%$ ), 5 to $25 \%$ immunopositivity (40\%) with moderate staining intensity $(86.7 \%)$ was observed in well-differentiated OSCC. Overall, there was a statistically significant difference among the three grades of OSCC in relation to survivin immunopositivity and immunoreactivity $(p<0.01)$.

Conclusion: This study supports the use of survivin as a potent diagnostic and prognostic marker for OSCC.

Clinical significance: Increased survivin expression and its nuclear localization appeared to correlate with a higher grade of malignancy suggesting unfavorable prognosis.

Keywords: Immunohistochemistry, Oral cancer, Prognostic marker, Survivin.

The Journal of Contemporary Dental Practice (2019): 10.5005/jp-journals-10024-2561
\end{abstract}

\section{INTRODUCTION}

Oral and pharyngeal cancer, grouped together, is the sixth most common cancer in the world. According to the International Agency for Research on Cancer of the World Health Organization (IARC-WHO), cancer rates are expected to increase at an alarming rate, from 10 million new cases globally in 2000 to 15 million in 2020. ${ }^{1}$ In the oral cavity, squamous cell carcinoma (SCC) is the most prevalent malignant neoplasm. The major risk factors for oral SCC (OSCC) are the use of tobacco or betel quid chewing, regular alcohol consumption, high-risk human papilloma virus (HR-HPV) infection, and diet containing lesser amounts of fresh fruits and vegetables. According to $\mathrm{WHO}, \mathrm{SCC}$ of head and neck can be graded based on epithelial differentiation into well, moderate, and poorly differentiated types. ${ }^{2}$

Survivin is one of the seven members of inhibitors of the apoptosis protein (IAP) family. The gene encoding human survivin was cloned by Ambrosini in 1997. All survivin isoforms contain one of the characteristic N-terminal BIR (Baculovirus IAP Repeats) domains and an alpha-helical region. The BIR domain is supposed to be important for antiapoptotic function, whereas the alpha helical domain interacts with tubulin structures. Survivin is expressed highly at the G2/M phase and declines rapidly in the G1 phase of the cell cycle. IAP family members inhibit apoptosis by inhibiting one or more caspases. Survivin is expressed diffusely during fetal development but is not generally found in normal adult tissues. It has been detected in various human cancers including bladder, colon, liver, brain, lung, and prostate and its expression levels correlate with their aggressiveness, implying poor prognosis and increased resistance to radiotherapy and chemotherapy. ${ }^{3}$ Nuclear survivin is assumed to control cell division, whereas cytoplasmic survivin is considered cytoprotective. Its selective
${ }^{1-3}$ Department of Oral Pathology and Microbiology, Best Dental Science College and Hospital, Madurai, Tamil Nadu, India

Corresponding Author: Ajithkumar M, Department of Oral Pathology and Microbiology, Best Dental Science College and Hospital, Madurai, Tamil Nadu, India, Phone: +91 8838262639, +91 8883445505, e-mail: ajithbds91@gmail.com

How to cite this article: Ajithkumar M, Murali CR, et al. Prognostic Implication of Survivin Expression in Oral Squamous Cell Carcinoma-An Immunohistochemical Study. J Contemp Dent Pract 2019;20(5):577-581.

Source of support: This research did not receive any specific grant from funding agencies in the public, commercial or not-for-profit sectors.

Conflict of interest: None

expression makes it a lead target for tumor diagnosis, prognosis, and anticancer therapies. Therefore, the present study aims to analyze the immunohistochemical expression of survivin in all the three histological grades of OSCC (well, moderate, and poorly differentiated OSCC) to evaluate its prognostic implication.

\section{Materials and Methods}

Prior to the commencement of the study, ethical approval was obtained from the institutional review board. This retrospective study was done by retrieving formalin-fixed paraffin-embedded tissue specimens (FFPE) of OSCC from the archives of Oral Pathology Department, Best Dental Science College \& Hospital, Madurai, India. The study material was composed of 60 FFPE tissue specimens that are divided into 4 groups: 15 cases each of well-differentiated (WDSCC), moderately differentiated (MDSCC), and poorly differentiated OSCC (PDSCC) and normal oral mucosal (NOM) tissue specimens.

(0) The Author(s). 2019 Open Access This article is distributed under the terms of the Creative Commons Attribution 4.0 International License (https://creativecommons. org/licenses/by-nc/4.0/), which permits unrestricted use, distribution, and non-commercial reproduction in any medium, provided you give appropriate credit to the original author(s) and the source, provide a link to the Creative Commons license, and indicate if changes were made. The Creative Commons Public Domain Dedication waiver (http://creativecommons.org/publicdomain/zero/1.0/) applies to the data made available in this article, unless otherwise stated. 


\section{Immunohistochemical Staining (IHC)}

Four micron thickness tissue sections were prepared and mounted on poly L-lysine-coated glass slides. Antigen retrieval was performed by heating the slides immersed in citrate buffer at $\mathrm{pH} 6$ in a microwave oven. Primary rabbit monoclonal antibodies against survivin (path in situ) were used. Biotinylated anti-rabbit IgG (Biogenex Supersensitive Detection system) was used as a secondary antibody and the bound antibody was detected using streptavidin-conjugated horseradish peroxidase (Biogenex Supersensitive Detection system) with 3,3'-diaminobenzidine as a substrate and Harris' hematoxylin as the counterstain. The high-grade human breast carcinoma specimen showing a strong expression for survivin was used as a positive control. Negative control was prepared by staining the positive control breast cancer specimen with the secondary antibody alone.

\section{Criteria for Evaluation}

Immunohistochemically stained slides were viewed by two observers using an Olympus BX 53 light microscope and compared with their respective $\mathrm{H}$ and $\mathrm{E}$ sections. Photomicrography was done with Prog Res Speed XT Core 3 software. Survivin immunopositivity was assessed by the presence of a brown color immunostaining of the nucleus and cytoplasm.

The intensity of staining was estimated based on the criteria followed by Nakagawa et al. ${ }^{4}$ : no staining (0); mild (1); moderate (2); and severe (3). The percentage of survivin immunopositivity was categorized based on the criteria adopted by Muzio et al. ${ }^{5}$ It was estimated and graded, in five random fields, on a scale of 0 to 4: $<5 \%$ immunopositive cells (0); 5 to $25 \%$ immunopositive cells (1); 26 to $50 \%$ immunopositive cells (2); $51 \%$ to $75 \%$ immunopositive cells (3); and $>75 \%$ immunopositive cells (4). The immunoreactivity of survivin was assessed semi-quantitatively by calculating the immunoreactive score (IRS) which is a product of the percentage of immunopositive cells and staining intensity. It was scored as negative (0-1); mild (2-3); moderate (4-8); and strongly positive (9-12).

\section{Statistical Methods}

Statistical analysis was done using Statistical Package for Social Sciences (SPSS Inc., Chicago, IL, version 22.0 for Windows). Fisher's Chi-square test was opted to compare the variance in distribution, intensity, percentage of immunopositivity, and also to assess pairwise comparison between the study groups. ANOVA was used to compare the immunoreactivity score of survivin within the four groups. " $p$ " value less than 0.05 denotes a significant relationship ( $p>0.05$-not significant, $p \leq 0.05$-significant, $p \leq 0.01$ - highly significant, and $p \leq 0.001$ - very highly significant).

\section{Results}

Survivin expression was found predominantly in the nuclear region of malignant epithelial cells in most cases of WDSCC $(66.7 \%)$ (Figs $1 \mathrm{~A}$ and B), MDSCC (60\%) (Figs $2 \mathrm{~A}$ and $\mathrm{B}$ ) and PDSCC (66.7\%) (Figs $3 A$ and $B$ ). Both cytoplasmic and nuclear expressions of survivin were found in $26.7 \%$ of each of WDSCC and MDSCC and $33.3 \%$ of PDSCC cases. However, cytoplasmic expression of this marker was observed only in few cases of WDSCC (6.7\%) and MDSCC (13.3\%). The intensity of the survivin reaction was found to be severe in $46.7 \%$ of PDSCC cases. Nevertheless, the majority of cases of WDSCC (86.7\%), MDSCC. (80\%), and PDSCC (53.3\%) showed moderate staining

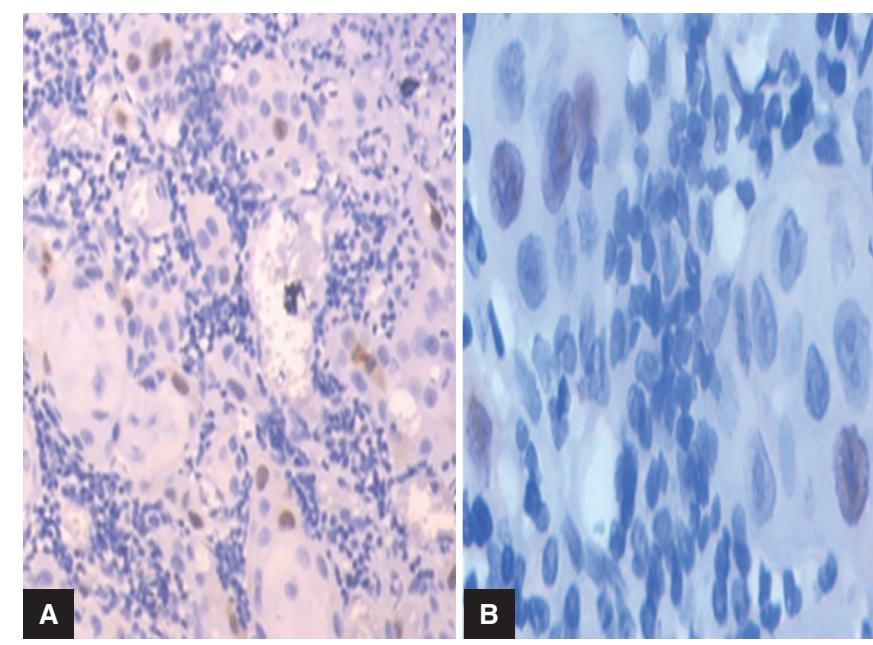

Figs $1 \mathrm{~A}$ and B: Mild immunopositivity for survivin in malignant epithelial cells in the nuclear region of WDSCC $[I H C, \times 100(A) ; \times 400$ (B)]

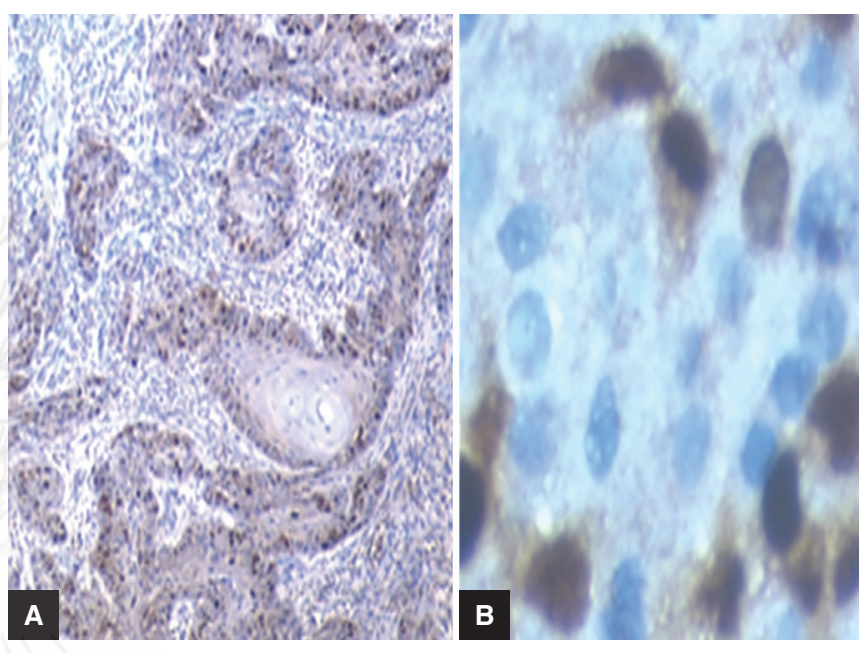

Figs $2 \mathrm{~A}$ and $\mathrm{B}$ : Moderate immunopositivity for survivin in malignant epithelial cells in the nuclear region of MDSCC $[I H C, \times 100(A) ; \times 400(B)]$

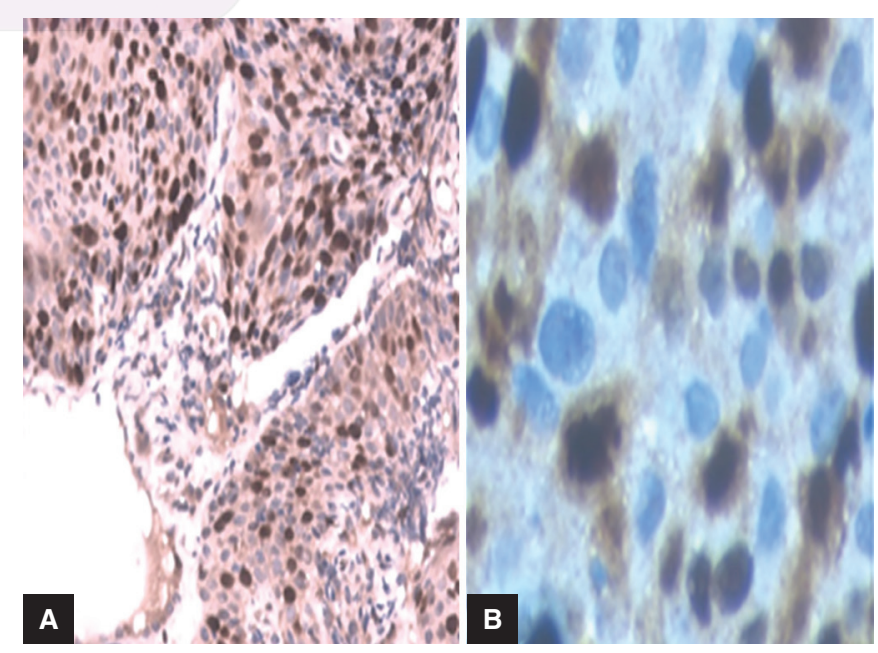

Figs 3A and B: Strong immunopositivity for survivin in malignant epithelial cells in the nuclear and few cytoplasmic regions of PDSCC [IHC, $\times 100(A) ; \times 400(B)]$ 


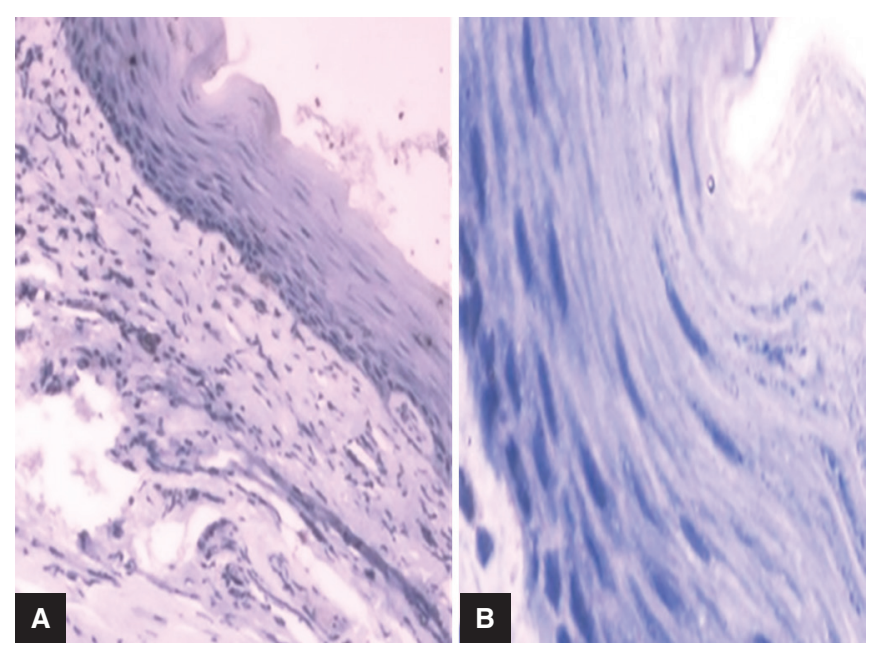

Figs 4A and B: Absence of survivin expression in NOM epithelium $[\mathrm{IHC}, \times 100(\mathrm{~A}) ; \times 400(\mathrm{~B})]$

intensity which was presented in Table 1. Survivin expression was totally absent in NOM epithelium (Figs 4A and B).

Most cases (40\%) of WDSCC had 5 to $25 \%$ immunopositive cells, while $33.3 \%$ of cases showed less than $5 \%$ positivity. However, the number of immunopositive cells was higher in MDSCC with around $40 \%$ of cases showing positivity in the range of 26 to $50 \%$ and $33.3 \%$ of samples with 51 to $75 \%$ immunopositivity. Comparatively, PDSCC exhibited greater percentage of survivin positivity with $53.3 \%$ of cases showing about 51 to $75 \%$ and $26.7 \%$ of samples with greater than $75 \%$ of positive cells (Table 2 ).

It was observed that the IRS for survivin increases with the grade OSCC. WDSCC showed mild (40\%) to moderate $(26.7 \%)$ immunoreactivity, whereas negative immunoreactivity was observed in $33.3 \%$ of cases. Most cases of MDSCC had moderate immunoreactivity (60\%) followed by mild (26.7\%) and strong (13.3\%) expressions. In the case of PDSCC, moderate (53.3\%) to strong immunoreactivity (46.7\%) was observed (Table 2 and Graph 1).

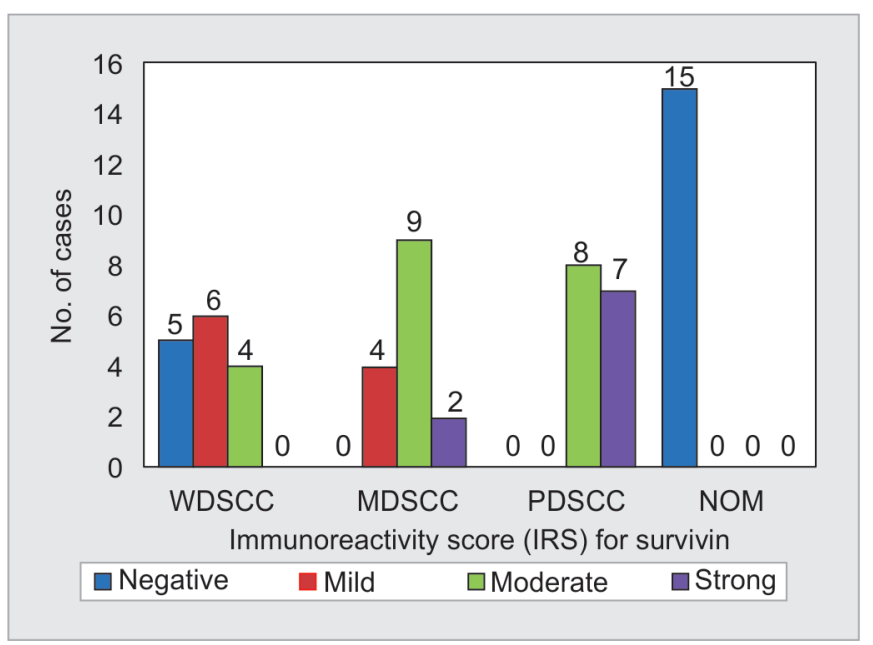

Graph 1: The comparison of the immunoreactivity score for survivin among the study groups

There exists a very high statistically significant difference in staining intensity and immunoreactivity of survivin among the study groups $(p<0.001)$. Pairwise comparison of survivin expression between the study groups is presented in Table 3.

\section{Discussion}

Several mechanisms have been proposed for the pathogenesis of OSCC. ${ }^{6-9}$ One of the mechanisms through which the tumor cells are believed to acquire resistance to apoptosis is by overexpression of the inhibitor of IAP. Survivin is one of the seven members of IAPs and its expression signals more aggressive and disseminated disease. Increased survivin expression is commonly associated with reduced levels of apoptosis and survival rate, increased proliferative index, recurrence rate, and resistance to chemotherapy and radiotherapy, thereby identifying it as a significant independent prognostic indicator of the poor outcome in patients with most tumor types.

Table 1: Comparison of immunohistochemical distribution and staining intensity of survivin among the study groups

\begin{tabular}{|c|c|c|c|c|c|c|c|c|}
\hline \multirow[b]{2}{*}{ Category } & \multicolumn{4}{|c|}{ Immunohistochemical distribution* } & \multicolumn{4}{|c|}{ Staining intensity* } \\
\hline & Absent & Cytoplasmic & Nuclear & $\begin{array}{l}\text { Cytoplasmic } \\
\text { and nuclear }\end{array}$ & Negative & Mild & Moderate & Severe \\
\hline WDSCC & $0(0 \%)$ & $1(6.7 \%)$ & $10(66.7 \%)$ & $4(26.6 \%)$ & $0(0 \%)$ & $0(0 \%)$ & $13(86.7 \%)$ & $2(13.3 \%)$ \\
\hline MDSCC & $0(0 \%)$ & $2(13.3 \%)$ & $9(60 \%)$ & $4(26.7 \%)$ & $0(0 \%)$ & $0(0 \%)$ & $12(80 \%)$ & $3(20 \%)$ \\
\hline PDSCC & $0(0 \%)$ & $0(0 \%)$ & $10(66.7 \%)$ & $5(33.3 \%)$ & $0(0 \%)$ & $0(0 \%)$ & $8(53.3 \%)$ & $7(46.7 \%)$ \\
\hline NOM & $15(100 \%)$ & $0(0 \%)$ & $0(0 \%)$ & $0(0 \%)$ & $15(100 \%)$ & $0(0 \%)$ & $0(0 \%)$ & $0(0 \%)$ \\
\hline$p$ value & $<0.001^{\dagger}$ & & & & $<0.001^{\dagger}$ & & & \\
\hline
\end{tabular}

${ }^{*}$ ANOVA test. ${ }^{\dagger} p$ value $<0.001$ (very highly significant)

Table 2: Comparison of immunopositivity and immunoreactivity scores of survivin among the study groups (WDSCC, MDSCC, PDSCC, and NOM)

\begin{tabular}{|c|c|c|c|c|c|c|c|c|c|}
\hline \multirow[b]{2}{*}{ Category } & \multicolumn{5}{|c|}{ Immunopositivity* } & \multicolumn{4}{|c|}{ Immunoreactivity score } \\
\hline & $<5 \%$ & $5-25 \%$ & $26-50 \%$ & $51-75 \%$ & $>75 \%$ & Negative & Mild & Moderate & Strong \\
\hline WDSCC & $5(33.3 \%)$ & $6(40 \%)$ & $4(26.7 \%)$ & $0(0 \%)$ & $0(0 \%)$ & $5(33.3 \%)$ & $6(40 \%)$ & $4(26.7 \%)$ & $0(0 \%)$ \\
\hline MDSCC & $0(0 \%)$ & $4(26.7 \%)$ & $6(40 \%)$ & $5(33.3 \%)$ & $0(0 \%)$ & $0(0 \%)$ & $4(26.7 \%)$ & $9(60 \%)$ & $2(13.3 \%)$ \\
\hline PDSCC & $0(0 \%)$ & $0(0 \%)$ & $3(20 \%)$ & $8(53.3 \%)$ & $4(26.7 \%)$ & $0(0 \%)$ & $0(0 \%)$ & $8(53.3 \%)$ & $7(46.7 \%)$ \\
\hline NOM & $15(100 \%)$ & $0(0 \%)$ & $0(0 \%)$ & $0(0 \%)$ & $0(0 \%)$ & $15(100 \%)$ & $0(0 \%)$ & $0(0 \%)$ & $0(0 \%)$ \\
\hline
\end{tabular}

${ }^{*}$ ANOVA test. ${ }^{\dagger} p$ value $<0.001$ (very highly significant) 
Survivin Expression in OSCC

Table 3: Pairwise comparison of survivin expression among the study groups

\begin{tabular}{|c|c|c|c|c|}
\hline Compared groups & Distribution $p$ value $e^{*}$ & Intensity $p$ value $e^{*}$ & Immunopositivity $p$ value $e^{*}$ & Immunoreactivity score $p$ value \\
\hline WDSCC vs MDSCC & 0.824 & 0.624 & $0.011^{\dagger}$ & $0.004^{\dagger}$ \\
\hline MDSCC vs PDSCC & 0.69 & 0.121 & $<0.001^{\dagger}$ & $0.002^{\dagger}$ \\
\hline WDSCC vs PDSCC & 0.69 & $0.046^{\dagger}$ & $<0.001^{\dagger}$ & $<0.001^{\dagger}$ \\
\hline
\end{tabular}

${ }^{*}$ Fischer's Chi-square test, ${ }^{\dagger} p$ value $\leq 0.05$ (statistically significant)

The immunopositivity for survivin was completely negative in normal oral epithelium, as observed in other studies by Gayathri et al., ${ }^{10}$ Lin et al., ${ }^{11}$ Khan et al., ${ }^{12}$ Li et al., ${ }^{13}$ and Jinbu et al. ${ }^{14}$ Survivin is generally expressed at high levels in embryonic tissues, but present at lower or non-detectable levels in normal adult tissues due to the presence of differentiated cells. ${ }^{11}$ In contrast to our findings, few studies have reported mild nuclear and cytoplasmic expression among sporadic cells of basal and parabasal layers. ${ }^{15,16}$ However, Negi et al. ${ }^{17}$ had observed survivin positivity in almost $20 \%$ of normal tissue specimens due to high proliferative capacity of the tissues. It was established that survivin immunoexpression in normal cells was due to the presence of active mitotic cells. ${ }^{18}$ Survivin was also shown to be present in normal hematopoietic cells, vascular endothelial cells, polymorphonuclear neutrophils, T lymphocytes, immune cells, melanocytes, keratinocytes, and neurons. ${ }^{19}$ Significant expression of survivin in normal oral epithelium can also occur due to processing errors during fixation, antigen retrieval techniques, types of primary antibodies, and detecting systems used. ${ }^{20}$

The present study reported a predominant nuclear positivity for survivin in all grades of OSCC though cytoplasmic and nuclear distribution was observed in few cases. Conversely, studies by Jinbu et al., ${ }^{14}$ Lippert et al., ${ }^{21}$ Li et al., ${ }^{22}$ and Pannone et al. ${ }^{23}$ have demonstrated greater expression of survivin in both cytoplasmic and nuclear regions of keratinocytes in OSCC. It was suggested that the nuclear pool of survivin is involved in promoting cell proliferation, ${ }^{23}$ whereas the cytoplasmic pool participates in controlling cell survival. ${ }^{10}$ The intensity of survivin expression was found to be moderate in most cases of WDSCC and MDSCC; nevertheless, PDSCC showed moderate to strong immunoreactivity, in concurrence with other studies by Gayathri et al. ${ }^{10}$ and Jane et al. ${ }^{24}$

A markedly increased expression of survivin was found in OSCC in comparison to normal oral epithelium, analogous to earlier studies by Preuss et al., ${ }^{25}$ Ciesielski et al.,r ${ }^{26}$ and Liping et al. ${ }^{27}$ This suggests that survivin can be a lead target for tumor diagnosis, prognosis, and anticancer therapies. Survivin was highly expressed in PDSCC compared to WDSCC and MDSCC which is statistically significant $(p<0.01)$. Though Muzio et al..$^{5}$ observed increased survivin expression in poorly differentiated OSCC, the differences were not statistically significant.

Further studies with larger sample size are required to assess the expression of survivin in OSCC cases to validate the significance of the study. Moreover, studies with clinical follow-up of patients are needed to evaluate the prognostic significance to confirm the role of survivin as a prognostic marker for OSCC. Survivin may act as a specific therapeutic target due to its unique expression in tumor cells and its absence in normal tissues. Various therapies involving survivin as a lead target are transcriptional inhibitors, small-molecule antagonists, Hsp90 inhibitors, cyclin-dependent kinase (CDK) inhibitors, promoter inhibitors, gene therapy, and immunotherapy. ${ }^{26}$ Transfection with dominant-negative mutants of survivin has led to increased apoptosis in gastric cancer cell lines and in breast cancer in animal models. ${ }^{28,29}$ Various survivin targeting immunotherapeutic strategies using survivin vaccines (SurVaxM) have been developed. ${ }^{30}$

\section{Conclusion}

Survivin expression and its nuclear localization appeared to correlate with a higher grade of malignancy. The greater immunoreactivity of survivin in poorly differentiated OSCC compared to well and moderately differentiated tumor suggests unfavorable prognosis. Based on the present study, it was concluded that survivin can be used as an important diagnostic and prognostic marker for an aggravated form of OSCC. To further validate survivin as a prognostic marker, a large-scale study with greater sample size along with clinical follow-up data is needed.

Due to its selective expression in tumor cells and absence in normal tissues, it has the potential to be used as a therapeutic target in various malignancies and is currently under phase I and II clinical trials. This might increase the effectiveness of radiation and chemotherapeutic agents in oral cancer patients and help in developing modified treatment strategies.

\section{ACKnOWLedgments}

Authors would like to thank Dr KS Prem Kumar (Principal, Best Dental Science College, Madurai) for consistent support throughout this project. Authors offer their deep gratitude to $\mathrm{Dr}$ Madhusudhanan and Mrs Manimegalai for helping them with the laboratory techniques and Mr K Aasaithambi (Biostatistician), who helped them with statistical analyses for the study. Authors would also like to thank Dr A Anjali, Dr M Rajanna Venkatraman, and Dr Antoinette Rhema Louis for their tremendous support to complete this research.

\section{References}

1. Warnakulasuriya S. Global epidemiology of oral and oropharyngeal cancer. Oral Oncol 2009 Apr 1;45(4):309-316. DOI: 10.1016/ j.oraloncology.2008.06.002.

2. Conceição Pereira M, Oliveira DT, et al. Histologic subtypes of oral squamous cell carcinoma: prognostic relevance. J Can Dent Assoc 2007 May 1;73(4):127-135.

3. Jaiswal PK, Goel A, et al. Survivin: a molecular biomarker in cancer. Ind J Med Res 2015 Apr;141(4):389-397. DOI: 10.4103/09715916.159250.

4. Nakagawa K, Yamamura K, et al. bcl-2 expression in epidermal keratinocytic diseases. Cancer 1994 Sep 15;74(6):1720-1724.

5. Muzio LL, Pannone G, et al. Survivin expression in oral squamous cell carcinoma. Br J Cancer 2003 Dec;89(12):2244-2248. DOI: 10.1038/ sj.bjc.6601402.

6. Rothenberg SM, Ellisen LW. The molecular pathogenesis of head and neck squamous cell carcinoma. J Clin Invest 2012 Jun 1;122(6):1951-1957.

7. Pérez-Sayáns M, Somoza-Martín JM, et al. Genetic and molecular alterations associated with oral squamous cell cancer. Oncol Rep 2009 Dec 1;22(6):1277-1282. 
8. Ram H, Sarkar J, et al. Oral Cancer: Risk Factors and Molecular Pathogenesis. J Maxillofac Oral Surg 2011 Apr;10(2):132-137. DOl: 10.1007/s12663-011-0195-z.

9. Williams HK. Molecular pathogenesis of oral squamous carcinoma. Mol Pathol 2000 Aug;53(4):165-172.

10. Gayathri C, Rao GV. Immunohistochemical expression of Survivin in oral leukoplakia and oral squamous cell carcinoma. J Univ Health Sci 2017 Jan;6:39-44.

11. Lin $\mathrm{CY}$, Hung $\mathrm{HC}$, et al. Survivin expression predicts poorer prognosis in patients with areca quid chewing-related oral squamous cell carcinoma in Taiwan. Oral Oncol 2005 Jul 1;41(6):645-654. DOI: 10.1016/j.oraloncology.2005.02.009.

12. Khan Z, Khan AA, et al. Survivin, a molecular target for therapeutic interventions in squamous cell carcinoma. Cell Mol Biol Lett 2017 Dec;22(1):8. DOI: 10.1186/s11658-017-0038-0.

13. Li SX, Chai L, et al. Expression of survivin and caspase 3 in oral squamous cell carcinoma and peritumoral tissue. Asian Pac J Cancer Prev 2012;13(10):5027-5031.

14. Jinbu $Y$, Tsukinoki $K$, et al. Expression of survivin in oral squamous cell carcinoma. Oral Med Pathol 2006 Jun 25;11(2):41-44.

15. Qi G, Kudo Y, et al. Nuclear Survivin expression is correlated with malignant behaviors of head and neck cancer together with Aurora-B. Oral Oncol 2010 Apr 1;46(4):263-270. DOI: 10.1016/j.oraloncology.2010.01.004.

16. Muzio LL, Campisi G, et al. HPV DNA and survivin expression in epithelial oral carcinogenesis: a relationship? Oral Oncol 2004 Aug 1;40(7):736-741. DOI: 10.1016/j.oraloncology.2003.11.011.

17. Negi A, Puri $A$, et al. Comparison of immunohistochemical expression of antiapoptotic protein survivin in normal oral mucosa, oral leukoplakia, and oral squamous cell carcinoma. Pathol Res Int 2015;2015:840739. DOI: 10.1155/2015/840739.

18. Tanaka C, Uzawa K, et al. Expression of an inhibitor of apoptosis, survivin, in oral carcinogenesis. J Dent Res 2003 Aug;82(8):607-611. DOI: $10.1177 / 154405910308200807$.

19. Fukuda S, Pelus LM. Survivin, a cancer target with an emerging role in normal adult tissues. Mol Cancer Ther 2006 May 1;5(5):1087-1098. DOI: 10.1158/1535-7163.MCT-05-0375.
20. Chaiyarit $P$, Jintakanon $D$, et al. Immunohistochemical analyses of survivin and heat shock protein 90 expression in patients with oral lichen planus. J Oral Pathol Med 2009 Jan;38(1):55-62. DOI: 10.1111/j.1600-0714.2008.00713.x.

21. Lippert BM, Knauer SK, et al. Dynamic survivin in head and neck cancer: molecular mechanism and therapeutic potential. Int J Cancer 2007 Sep 15;121(6):1169-1174. DOI: 10.1002/ijc.22941.

22. Li F, Yang J, et al. Nuclear or cytoplasmic expression of survivin: what is the significance? Int J Cancer 2005 Apr 20;114(4):509-512. DOI: 10.1002/ijc.20768.

23. Pannone G, Bufo $P$, et al. Survivin phosphorylation and M-phase promoting factor in oral carcinogenesis. Histol Histopathol $2007 \mathrm{Nov}$ 1;22(10/12):1241-1249. DOI: 10.14670/HH-22.1241.

24. Jane C, Nerurkar AV, et al. Increased survivin expression in high-grade oral squamous cell carcinoma: a study in Indian tobacco chewers. J Oral Pathol Med 2006 Nov;35(10):595-601. DOI: 10.1111/j.1600 0714.2006.00473.x.

25. Preuss SF, Weinell A, et al. Nuclear survivin expression is associated with HPV-independent carcinogenesis and is an indicator of poor prognosis in oropharyngeal cancer. Br J Cancer 2008 Feb;98(3): 627-632. DOI: 10.1038/sj.bjc.6604192.

26. Ciesielski MJ, Qiu J, et al. Survivin as a cancer vaccine target. J Vaccines Vaccin 2014 May;5(3):230-236.

27. Liping $S$, Ying $W$, et al. Up-regulation of survivin in oral squamous cell carcinoma correlates with poor prognosis and chemoresistance. Oral Surg, Oral Med, Oral Pathol, Oral Radiol Endodontol 2010 Oct 1;110(4): 484-491. DOI: 10.1016/j.tripleo.2010.04.009.

28. Tu SP, Jiang $\mathrm{XH}$, et al. Suppression of survivin expression inhibits in vivo tumorigenicity and angiogenesis in gastric cancer. Cancer Res 2003 Nov 15;63(22):7724-7732.

29. Mesri $M$, Wall NR, et al. Cancer gene therapy using a survivin mutant adenovirus. J Clin Invest 2001 Oct 1;108(7):981-990. DOI: 10.1172/ JCl12983.

30. Mobahat M, Narendran A, et al. Survivin as a Preferential Target for Cancer Therapy. Int J Mol Sci 2014 Feb 13;15(2):2494-2516. DOI: 10.3390/ijms15022494. 